\title{
Time and Temperature are of the Essence in Kidney
}

\author{
Khan $\mathrm{T}^{1}$, Kwarcinski $\mathrm{J}^{1}$, Robertson $\mathrm{H}^{1}$, Robertson $\mathrm{P}^{1}$, Hameed $\mathrm{A}^{1}$, Yoon $\mathrm{P}^{1}$, Lee $\mathrm{T}^{1}$, Yuen $\mathrm{L}^{1}$, \\ Laurence JM${ }^{1}$, Pang $\mathrm{T}^{1}$, Rogers $\mathrm{N}^{2}$, Wong $\mathrm{G}^{2}$, Hawthorne $\mathrm{W}^{1,2}$ and Henry C Pleass ${ }^{1,2 *}$ \\ ${ }^{1}$ Department of Surgery, University of Sydney, Australia
}

${ }^{2}$ Department of Renal Medicine, University of Sydney, Australia

*Corresponding author: Henry C Pleass, Department of Surgery, University of Sydney, Australia.

To Cite This Article: Khan T, Kwarcinski J, Robertson H, Robertson P, Henry C Pleass, et al., Time and Temperature are of the Essence in Kidney Transplantation. Am J Biomed Sci \& Res. 2021 - 14(3). AJBSR.MS.ID.001991. DOI: 10.34297/AJBSR.2021.14.001991.

Received: 留September 25, 2021; Published: 眥 October 04, 2021

\section{Introduction}

Kidney transplantation has become a routine and effective surgical procedure since the first successful transplant in 1954 [1]. Over the last 40 years, much attention has focussed on the development of immunosuppression to improve patient outcomes. Kidney transplantation, for suitable patients, provides benefits both in duration and quality of life when compared with other forms of kidney replacement therapy [2]. However, the number of suitable recipients far exceeds the supply of appropriate donor organs worldwide. In Australia and New Zealand, less than $9 \%$ of dialysis patients are ever listed for kidney transplantation, meaning the majority will die on dialysis. This organ shortage has stimulated interest in both increasing the number of suitable organ donors, as well as improving more marginal organs that are currently being utilised for transplantation [2-7]. The majority of deceased donor kidneys are retrieved from patients who typically had significant co-morbidities prior to their death, as well as age-related changes that affect their renal function and resilience to further ischaemic injury. Deceased donation follows either a donation after brain death (DBD) pathway or a donation after circulatory death (DCD) pathway. The DCD pathway accounts for 30 to $40 \%$ of the deceased donor program in many kidneys transplant programs $[3,4,8]$. These DCD kidneys generally suffer a greater ischemia-reperfusion injury than DBD organs and have a significantly higher risk of delayed graft function (DGF), as well as primary non function, when compared with their DBD pathway counterparts. An organ deprived of the supply of oxygenated blood, sustains damage in a manner proportional to the length of the anoxic exposure and its temperature during this time. Following organ retrieval, there is a period of storage often for transportation, the so-called cold ischemic time (CIT). CIT impacts both delayed graft function and graft survival and is kept to a minimum but is highly dependent on logistics [9]. DCD organs suffer an additional ischaemic hit in the form of the warm ischaemic time (WIT), which is variably defined but generally denoted by the period between cessation of circulation and the commencement of cold in situ perfusion. Machine perfusion technology, including hypothermic and normothermic, has shown promise in rejuvenating kidneys following storage and transportation and is the subject of ongoing active research worldwide [10]. During the recipient operation, the time between removing the kidney from ice to revascularization with recipient blood is called the second warm ischaemic time (SWIT). There is a clear association between increasing WIT and the incidence of delayed graft function, biopsy-proven organ injury graft failure and patient death [11-13]. The impact of WIT is more pronounced in kidneys from DCD donors within the latter US cohort, although it negatively impacted all deceased donor kidneys. Whilst every effort is made to minimise the WIT during implantation, relatively little attention has been paid to the warm ischaemic associated with deceased organ donor retrieval, likely as it is perceived as largely unmodifiable. However, this ischaemic time does have an impact on outcome and is modifiable. In a recently published European study, the time taken from kidney perfusion to removal and placement on ice was an independent predictor of death-censored graft failure in DCD, but not DBD kidneys [14]. Simply, for every additional 10 mins to placing the kidney on ice, there was a $5 \%$ increased risk of graft failure. Our own group has determined similar issues in donor organ function related to surgical operating time in the deceased donor procedure. To differentiate this ischaemic time from CIT 
and WIT we termed it the "donor in situ ischaemic time" (DISIT). [15] The DISIT has been shown to be an independent risk factor for delayed graft function in our single centre cohort. The impacts of DISIT on kidney and liver graft function [16] are being increasingly appreciated in the literature, although they have not yet attracted much recognition from donor retrieval services worldwide.

The starting temperature of the kidney stored on ice is $1-4^{\circ} \mathrm{C}$. The kidney rewarms at approximately $0.5^{\circ} / \mathrm{min}$ and its final temperature is therefore dependant on vascular anastomosis time [17]. Techniques to slow rewarming of the kidney, often termed "regional hypothermia", have been in routine use in urological surgery for more than half a century [18]. The concept was described in animal models for kidney transplantation in the 1990's [19], and subsequently adopted into small clinical studies [20]. These data suggest that significant short-term damage to the graft is avoided if kidney temperature remains below $15-18^{\circ} \mathrm{C}$. Therefore, kidney cooling may offer some advantages even in routine clinical practice, although it is not widely used because it is cumbersome to arrange as a routine. We have reproduced the temperature rise of human kidneys, both in an ex vivo water bath model of transplantation (Figure 1), in addition to intra-operative temperature measurements in clinical kidney transplant procedures. Even with relatively short SWIT, the temperature threshold for organ injury (of approximately $150^{\circ} \mathrm{C}$ ) is breached and so we have developed an insulating jacket, designed to fit all adult sized kidneys. We have proven thermal protection using this jacket in a porcine kidney ex vivo model [21]. This has now been validated with discarded human kidneys and again shows prolonged protection against organ temperature rise in an ex vivo model of kidney transplantation. While additional research is required to determine the long-term effects of thermal insulation on the kidney allograft, including delayed graft function and allograft survival, the results of this experiment highlight that SWIT can be mitigated. By using thermal insulating jackets, the time pressures associated with kidney transplantation are reduced, minimising risks of surgical complication and expanding pathways for surgical training and facilitating robotic transplantation [21]. In conclusion, time and temperature are very much of the essence in kidney transplantation. Retrieval surgical teams need both the understanding and skills to limit the donor in situ ischaemic time for all organs. The subsequent temperature rises and organ injury that occurs during kidney implantation, we believe, can be limited with the aid of an insulating device, to both reduce the incidence of delayed graft function as well as improve long-term graft survival. This will have major implications for both the training of transplant surgeons, as well as the introduction of robotic surgery to the broader transplant community.

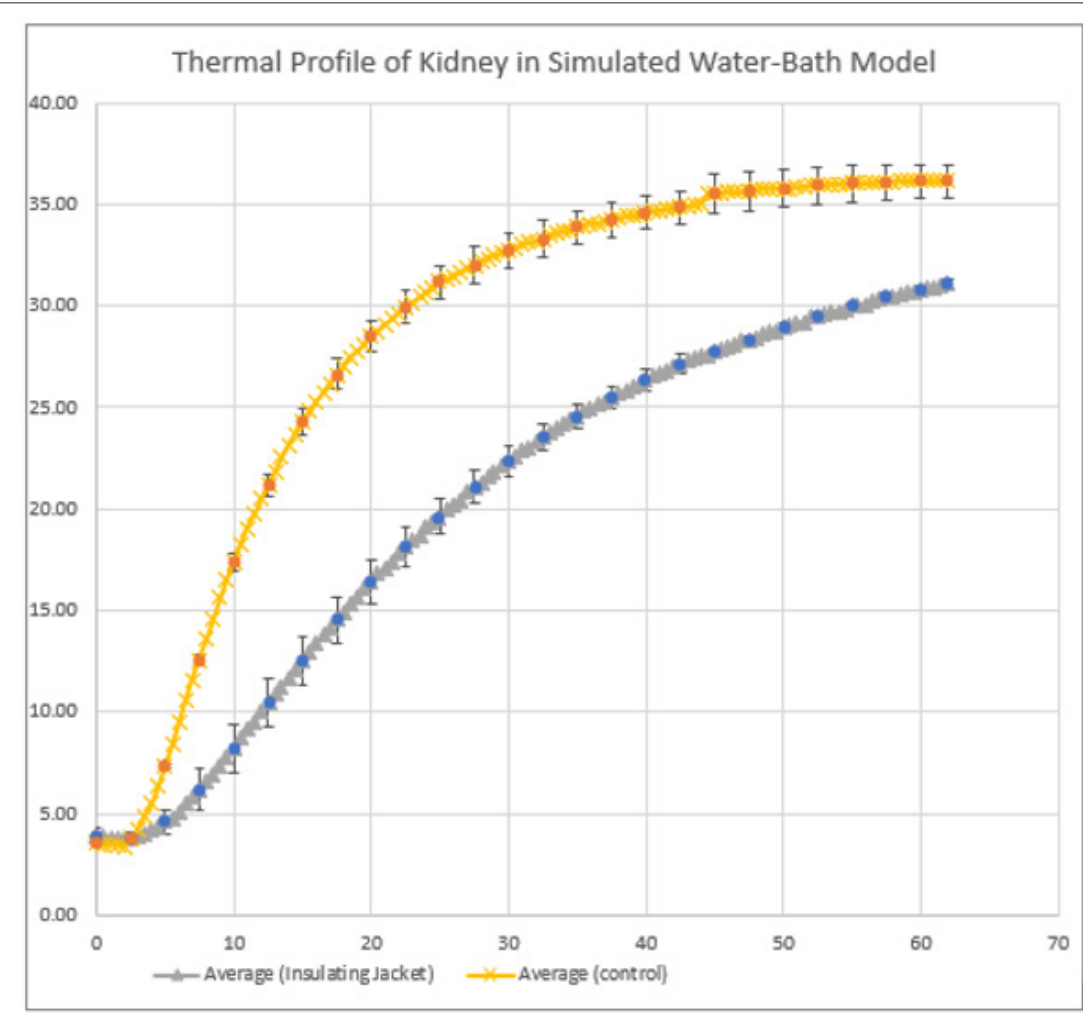

Figure 1: Water Bath Model of Transplantation. 


\section{References}

1. RA Wolfe, VB Ashby, EL Milford, AO Ojo, RE Ettenger, et al. (1999) Comparison of mortality in all patients on dialysis, patients on dialysis awaiting transplantation and recipients of a first cadaveric transplantation. N Engl J Med 341(23): 1725-1730.

2. Stephen P McDonald (2015) Australia and New Zealand Dialysis and Transplant Registry. Kidney Int Suppl 5(1): 39-44.

3. R Hodgson, AL Young, MA Attia, JPA Lodge (2017) Impact of a National Controlled Donation After Circulatory Death (DCD) Program on Organ Donation in the United Kingdom: A 10-Year Study. Am J Transplant 17(12): 3172-3182.

4. John Gill, Caren Rose, Julie Lesage, Yayuk Joffres, Jagbir Gill, et al. (2017) Use and Outcomes of Kidneys from Donation after Circulatory Death Donors in the United States. J Am Soc Nephrol 28(12): 3647-3657.

5. Karthik K Tennankore, S Joseph Kim, Ian PJ Alwayn, Bryce A Kiberd (2016) Prolonged warm ischemia time is associated with graft failure and mortality after kidney transplantation. Kidney Int 89(3): 648-658.

6. H Peters-Sengers, JHE Houtzager, MBA Heemskerk, MM Idu, RC Minnee, et al. (2018) DCD donor hemodynamics as predictor of outcome after kidney transplantation. Am J Transplant 18(8): 1966-1976.

7. MB Allen, E Billig, PP Reese, J Shults, R Hasz, et al. (2016) Donor Hemodynamics as a Predictor of Outcomes After Kidney Transplantation from Donors After Cardiac Death. Am J Transplant 16(1): 181-193.

8. Chris J Callaghan, Simon JF Harper, Kourosh Saeb-Parsy, Alex Hudson, Paul Gibbs, et al. (2014) The discard of deceased donor kidneys in the UK. Clin Transplant 28(3): 345-353.

9. Claudio E Ponticelli (2015) The impact of cold ischemia time on renal transplant outcome. Kidney international 87(2): 272-275.

10. Ahmer M Hameed, Henry C Pleass, Germaine Wong, Wayne J Hawthorne (2016) Maximizing kidneys for transplantation using machine perfusion: from the past to the future: A comprehensive systematic review and meta-analysis. Medicine 95(40): 5083.

11. L Heylen, M Naesens, I Jochmans, D Monbaliu, E Lerut, et al. (2015) The effect of anastomosis time on outcome in recipients of kidneys donated after brain death: a cohort study. American Journal of Transplantation 15(11): 2900-2907.
12. L Heylen, J Pirenne, U Samuel, I Tieken, M Naesens, et al. (2017) The impact of anastomosis time during kidney transplantation on graft loss: a Eurotransplant cohort study. American Journal of transplantation 17(3): 724-732.

13. Karthik K Tennankore, S Joseph Kim, Ian PJ Alwayn, Bryce A Kiberd (2016) Prolonged warm ischemia time is associated with graft failure and mortality after kidney transplantation. Kidney International 89(3): 648-658.

14. L Heylen, J Pirenne, U Samuel, I Tieken, M Coemans, et al. (2020) Effect of donor nephrectomy time during circulatory-dead donor kidney retrieval on transplant graft failure. Journal of British Surgery 107(1): 87-95.

15. (2021) Abstracts for the 2021 Transplantation Society of Australia and New Zealand Annual Scientific Meeting. Transplantation Direct 7(5): $1-52$.

16. Dieter Adelmann, Garrett R Roll, Rishi Kothari, Shareef Syed, Lyle J Burdine, et al. (2018) The impact of deceased donor liver extraction time on early allograft function in adult liver transplant recipients. Transplantation 102(11): 466-471.

17. Benoît Feuillu, Luc Cormier, Luc Frimat, Michele Kessler, Mohamed Amrani, et al. (2003) Kidney warming during transplantation. Transpl Int 16(5): 307-312.

18. JE Wickham (1971) Regional renal hypothermia. Ann R Coll Surg Engl 48(2): 99-113.

19. JL R Forsythe, PM Dunnigan, G Proud, TWJ Lennard and RMR Taylor (1989) Reducing renal injury during transplantation. Br J Surg 76(10): 999-1001.

20. M Szostek, M Pacholczyk, B Lagiewska, R Danielewicz, J Wałaszwski, et al. (1996) Effective surface cooling of the kidney during vascular anastomosis decreases the risk of delayed kidney function after transplantation. Transpl Int 9(1): 84-85.

21. Turaab Khan, Jeremy Kwarcinski, Tony Pang, Ahmer Hameed, Philip Boughton, et al. (2021) Protection From the Second Warm Ischemic Injury in Kidney Transplantation Using an Ex Vivo Porcine Model and Thermally Insulating Jackets in Transplantation Proceedings. Elsevier 53(2): 750-754. 